\title{
Modular Invariants in the Fractional Quantum Hall Effect
}

\author{
KAZUSUMI INO \\ Institute for Solid State Physics, University of Tokyo, \\ Roppongi 7-22-1, Minatoku, Tokyo, 106, Japan \\ ino@kodama.issp.u-tokyo.ac.jp
}

\begin{abstract}
We investigate the modular properties of the characters which appear in the partition functions of nonabelian fractional quantum Hall states. We first give the annulus partition function for nonabelian FQH states formed by spinon and holon (spinon-holon state). The degrees of freedom of spin are described by the affine $S U(2)$ Kac-Moody algebra at level $k$. The partition function and the Hilbert space of the edge excitations decomposed differently according to whether $k$ is even or odd. We then investigate the full modular properties of the extended characters for nonabelian fractional quantum Hall states. We explicitly verify the modular invariance of the annulus grand partition functions for spinon-holon states, the Pfaffian state and the 331 states. This enables one to extend the relation between the modular behavior and the topological order to nonabelian cases. For the Haldane-Rezayi state, we find that the extended characters do not form a representation of the modular group, thus the modular invariance is broken. We also find a new relation between the Haldane-Rezayi state and the 331 state and suggest its implications for 'The $\nu=\frac{5}{2}$ Enigma'.
\end{abstract}




\section{Introduction}

Fractional quantum Hall (FQH) states [1, 2] have edge excitations as their low energy excitations [3, 4]. Some experiments have already observed characteristic behaviors of theoretical predictions [5, 6]. The edge excitations form representations of chiral algebra extended by the holomorphic field for the electron. One way to give a complete description of the Hilbert space of edge excitations is to construct the partition function. The partition function for edge excitations has been discussed for a disk and an annulus geometry [4, 7, 8, 9, 10]. Especially, Cappelli and Zemba [10] gave extended modular invariance conditions which should be satisfied by the annulus partition function for edge excitations of abelian quantum Hall states. The annulus partition function encodes not only the spectrum of edge excitations but also the topological order [11] in its modular properties through the Verlinde formula. They also found non-diagonal modular invariants partition functions for abelian cases from the charcters of the affine $U(1)^{m}$ algebra, which may explain the experimentally observed plateaus beyond the Jain's series [12] such as $\nu=\frac{4}{11}, \frac{4}{13}$. Recently all the possible $U(1)^{m}$ modular invariants for abelian quantum Hall effect were classified by Gannon [13]. Also the annulus partition functions for the paired states (Pfaffian [14, Haldane-Rezayi [15]) were derived in [9], but the modular properties of these partition functions have not been worked out.

In this paper, we first derive the partition functions for the edge excitations of the spinsinglet FQH state (spinon-holon state) constructed from conformal blocks of the $S U(2)$ Wess-Zumino-Witten model at level $k$. This state is an example of nonabelian FQH states 14] when $k>1$. The statistical property of the quasiparticle in this state was discussed by Blok and Wen [16]. The case of $k=1$ arises from the Jain's hierarchy [17, 18, 10, 19]. It also gives the spin-singlet Halperin state [20, 21]. In these models, electrons or composite fermions form a spin $k / 2$ representation of $S U(2)$. The degeneracy may arise from spin, multi-layer, degenerate bands of composite fermions and so on. We examine the edge excitations of spinon-holon states from the bulk wave functions obtained from the bulk CFT [14, 22], which is based on the general relation between the bulk CFT and Chern-Simons theory [23]. According to that, edge states can be treated like states on punctures in the thermodyamic limit, which is also supported by a numerical study [8]. We find that the partition function or the Hilbert space of the edge excitations is decomposed differently according to whether $k$ is even or odd. Accordingly, the bulk excitation of the state have the different quasiparticle spectrum in the holon's part.

We then investigate the modular properties of the characters which appear in annulus partition functions for nonabelian FQH states including the spinon-holon states and the paired states (Pfaffian, Haldane-Rezayi, 331). These states are of great interest since they may be realized in the even-denominator plateaus in a single and double-layers systems [24, 25, 26]. Among them, the $\nu=\frac{5}{2}$ plateau [24] remains an 'enigma' [27]. Experiments support that the state is the spin-singlet Haldane-Rezayi state. Numerical studies, however, show that the spin-polarized Pfaffian-like state is more favored [26]. Recently nonabelian statistics in these paired states have attracted considerable attentions [28, 29, 30, 31]. Although the modular properties of the Haldane-Rezayi state are discussed in Ref.[29], the full modular properties including the charge sectors of nonabelian FQH states have not been addressed. We derive the full modular matrix elements of extended characters and explicitly verify the extended modular invariance conditions for spinon-holon states, the Pfaffian states and the 331 states. For the Haldane-Rezayi state, we find that the extended characters do not form a representation of the modular group. This implies that the annulus partition functions 
cannot be modular invariant and requires some modification. We give a new way to cure this pathologic behavior by establishing new relation between the Haldane-Rezayi state and the 331 state.

For the other nonabelian FQH states, the modular invariance enables one to extend the relation between the topological order and modular properties of Cappelli and Zemba.

The organization of the paper is as follows. In Section 2, we derive the partition functions for the spinon-holon FQH state using the method of Ref. 33. In Sec. 3, we determine the modular properties of the characters of nonabelian FQH states, and investigate the extended modular invariance conditions for them. Sec. 4 is devoted to conclusions.

\section{Spinon-Holon FQH States}

We consider the FQH states formed by spinon and holon. The degrees of freedom of spin is carried by spinon, given by the affine $S U(2)_{k}$ algebra. The degrees of freedom of holon is carried by holon, given by the affine $U(1)$ Kac-Moody algebra.

Let us first recall the representation of $S U(2)_{k}$. $S U(2)_{k}$ has $k+1$ integrable representations $\left[\phi_{l}\right]$ with $l=0, \cdots, k$, namely the ones with $S U(2)$ isospin $\frac{1}{2} l \leq \frac{1}{2} k$. The conformal weight for $\phi_{l}$ is

$$
h_{l}=\frac{l(l+2)}{4(k+2)}
$$

The fusion rules are 37

$$
\phi_{l} \times \phi_{l^{\prime}}=\sum_{j=\left|l-l^{\prime}\right|}^{\min \left(l+l^{\prime}, 2 k-l-l^{\prime}\right)} \phi_{j},
$$

where $j-\left|l-l^{\prime}\right|$ is an even integer. This model is supposed to have a quasiparticle with nonabelian statistics when $k>1$ [16].

Disk We first consider the edge excitations for a disk geometry. We can form the groundstate wave function from the primary fields in $S U(2)_{k}$ by coupling a chiral boson $\varphi$. Since the electrons must have fermionic statistics, they are constructed from a so-called simple current i.e. whose operator product expansion with itself only gives rise to one representation. From (2.2), the only simple current in this model is the representation $\left[\phi_{k}\right]$. Since $\phi_{k}$ has a conformal weight $h_{k}=\frac{k}{4}$, one couples the field $e^{i \sqrt{q} \varphi}$ with $q=\frac{k}{2}+s$ to form a well defined wave function. Here $s+k$ must be an odd integer to account for the fermionic statistics of electron. This can be seen by using fermionic construction of $S U(2)_{k}$ WZW model [16]. The primary field for the electron is then,

$$
V_{k}^{\alpha} e^{i \sqrt{q} \varphi}
$$

where $V_{k}^{\alpha}, \quad \alpha=-k,-k+1, \cdots, k$ are the vertex operators of $\left[\phi_{k}\right]$. We can also take $e^{-i \sqrt{q} \varphi}$ in (2.3). In that case, we refer to the number of electrons as $-N$ and $\varphi$ would be replaced by $-\varphi$ in the following. From these operators, we can construct the ground-state wave function for a fractional quantum Hall state of $N S U(2)$ electrons on a disk:

$$
\left\langle\Psi_{\text {edge }}^{\vee}\left|\prod_{i=1}^{N} V_{k}^{\alpha_{i}} e^{i \sqrt{q} \varphi}\right| v a c\right\rangle \exp \left[\sum_{i}-\frac{1}{4}\left|z_{i}\right|^{2}\right],
$$


where $\alpha_{1}, \cdots \alpha_{N}$ are indices for the spin of spinons and $\Psi_{\text {edge }}$ is given by

$$
\begin{aligned}
\Psi_{\text {edge }} & =e^{-i N \sqrt{q} \varphi}, \quad h_{\text {edge }}=\frac{1}{2} N^{2} q . \quad \text { for } N \text { even, } \\
\Psi_{\text {edge }} & =V_{k}^{\alpha_{\infty}} e^{-i N \sqrt{q} \varphi}, \quad h_{\text {edge }}=\frac{k}{4}+\frac{1}{2} N^{2} q, \quad \text { for } N \text { odd }, \\
\left\langle\Psi_{\text {edge }}^{\vee}\right| & =\lim _{z_{\infty} \rightarrow \infty}\langle v a c| \Psi_{\text {edge }}\left(z_{\infty}\right) z_{\infty}^{2 h_{\text {edge }}} .
\end{aligned}
$$

This state has a filling fraction $\nu=\frac{1}{q}$.

Let us especially consider $S U(2)_{1}$. $S U(2)_{1}$ WZW model has two primary fields,

$$
V^{\downarrow}\left(z_{\downarrow}\right), \quad V^{\uparrow}\left(z_{\uparrow}\right) .
$$

The conformal weights for these vertex operators are $\frac{1}{4}$. We can construct a $S U(2)$ singlet ground state from these fields ( $N$ :even):

$$
\left\langle\prod_{i=1}^{N} V^{\uparrow}\left(z_{i}^{\uparrow}\right) e^{i \sqrt{n+\frac{1}{2}} \varphi\left(z_{i}^{\uparrow}\right)} \prod_{i=1}^{N} V^{\downarrow}\left(z_{i}^{\downarrow}\right) e^{i \sqrt{n+\frac{1}{2}} \varphi\left(z_{i}^{\downarrow}\right)}\right\rangle .
$$

This wave function is manifestly $S U(2)$ invariant. It is calculated to be

$$
\Phi_{\text {Halp }}=\prod_{i<j}\left(z_{i}^{\uparrow}-z_{j}^{\uparrow}\right)^{n+1} \prod_{i<j}\left(z_{i}^{\downarrow}-z_{j}^{\downarrow}\right)^{n+1} \prod_{i<j}\left(z_{i}^{\uparrow}-z_{j}^{\downarrow}\right)^{n} \exp \left[-\frac{1}{4} \sum\left(\left|z_{i}^{\uparrow}\right|^{2}+\left|z_{i}^{\downarrow}\right|^{2}\right)\right] .
$$

This is the spin-singlet state called the Halperin state [20]. In this case, the spinon is semion.

For general $k$, the spectrum of excitations is restricted by the requirement of singlevaluedness and non-singularity of wave function in electron coordinates. Since $V_{j}^{\alpha_{1}}(j=$ $1, \cdots, k)$ and $V_{k}^{\alpha_{2}}$ have an operator product expansion

$$
V_{j}^{\alpha_{1}}\left(z_{1}\right) V_{k}^{\alpha_{2}}\left(z_{2}\right) \sim \frac{C_{\alpha_{1} \alpha_{2}}^{\alpha}}{\left(z_{1}-z_{2}\right)^{\frac{j}{2}}} V_{k-j}^{\alpha}\left(z_{2}\right)+\cdots
$$

where $C_{\alpha_{1} \alpha_{2}}^{\alpha}$ is a constant, the allowed couplings are

$$
\begin{gathered}
V_{j} e^{i \frac{(2 n+j)}{2 \sqrt{q}} \varphi}, \quad n \in \mathbf{N}, \text { for } j: \text { odd } \\
V_{j} e^{i \frac{n+j / 2}{\sqrt{q}} \varphi}, n \in \mathbf{N}, \text { for } j: \text { even. }
\end{gathered}
$$

To see the relation between the bulk and the edge explicitly, let us use the chiral vertex operator (CVO) expression of FQH state [33]. We denote the CVO : $\left[\phi_{j_{3}}\right] \rightarrow \operatorname{Hom}\left(\left[\phi_{j_{1}}\right] \rightarrow\right.$ $\left.\left[\phi_{j_{2}}\right]\right)$ for $S U(2)_{k}$ WZW model as

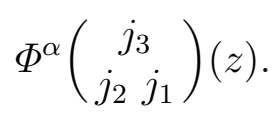

For our purpose, the CVO represents the annulus with the representation $\left[\phi_{j_{1}}\right]$ inserted at $z$ with the inner and outer edge states $\left[\phi_{j_{2}}\right]$ and $\left[\phi_{j_{3}}{ }^{\mathrm{V}}\right]$ respectively [33.

Suppose that we take a disk-like region $D_{1}$ in the disk, which contains only one electron. Then we make a series of annulus-like regions $D_{2} \cdots D_{N}$, each of which contains one electron respectively such that the outer region of $D_{N}$ is the boundary of the disk. One can correspond 
$D_{1}, \cdots D_{N}$ to CVOs. Then the ground-state wave function (2.4) can be expressed as, for even $N$ :

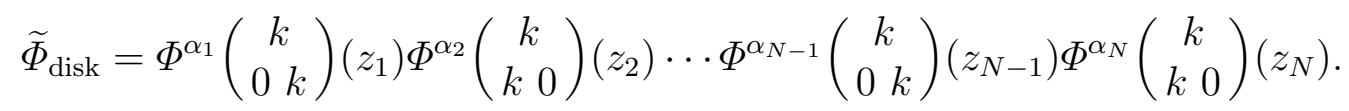

for odd $N$ :

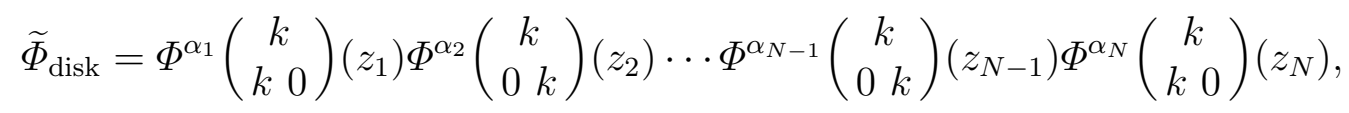

Here we have omitted the indices for the rational torus.

The edge excitations are generated on the field at infinity, $\Psi_{\text {edge }}$. It is $V_{0}^{\alpha_{\infty}}$ for $N$ even and $V_{k}^{\alpha_{\infty}}$ for $N$ odd. Edge excitations are generated by current algebras as

$$
J_{-n_{1}}^{a_{1}} \cdots J_{-n_{l_{1}}}^{a_{l_{1}}} \widetilde{j}_{-n_{1}^{\prime}} \cdots \widetilde{j}_{-n_{l_{2}}^{\prime}} \Psi_{\text {edge }}
$$

where $\widetilde{j_{n}}$ is the generator of the affine $U(1)$ Kac-Moody algebra

$$
\left[\widetilde{j}_{n}, \widetilde{j}_{m}\right]=n \delta_{m+n}
$$

and $J_{n}^{a}$ are the generators of the affine $S U(2)_{k}$ Kac-Moody algebra satisfying

$$
\left[J_{m}^{a}, J_{n}^{b}\right]=i \epsilon^{a b c} J_{m+n}^{c}+k m \delta^{a b} \delta_{m+n, 0} .
$$

Here $\epsilon^{a b c}$ is a rank 3 antisymmetric tensor. We denote CVO expressions for the edge excited state as $\widetilde{\Phi}^{\left(n_{1}, n_{2}, \cdots\right)}$.

We define an operator $M$ on chiral vertex operators by

$$
M \Phi^{\alpha}\left(\begin{array}{c}
j_{3} \\
j_{2} j_{1}
\end{array}\right)=\left(-\Delta_{j_{1}}+\Delta_{j_{3}}-\Delta_{j_{2}}\right) \Phi^{\alpha}\left(\begin{array}{c}
j_{3} \\
j_{2} j_{1}
\end{array}\right)(z)
$$

where $\Delta_{j_{m}}$ is the conformal weight of the field $\phi_{j_{m}}$. The action on the expressions with descendant field is defined similarly. We demand that it satisfies the Leibnitz rule when it acts on the product of chiral vertex operators. As shown in [33], $M$ acts as the angularmomentum operator on these states when it acts on the monomials $\widetilde{\Phi}^{\left(n_{1}, n_{2}, \cdots\right)}$. Let us denote the vector space spanned by such monomials as $\Omega_{\text {edge }}$. Then the partition function for the state on the disk is given by

$$
\begin{aligned}
Z^{\text {disk }}(\tau)=\operatorname{Tr}_{\Omega_{\text {edge }}}\left(\mathrm{e}^{2 \pi i \tau\left(M-\frac{c}{24}\right)}\right) & =\frac{\omega^{M_{0}} \chi_{0}^{(k)}(\tau)}{\eta(\tau)} \text { for } N \text { even, } \\
M_{0} & =\frac{1}{2} q N(N-1)-\frac{k}{4} N \\
Z^{\text {disk }}(\tau) & =\frac{\omega^{M_{0}} \chi_{k}^{(k)}(\tau)}{\eta(\tau)} \text { for } N \text { odd } \\
c=\frac{3 k}{k+2}+1, \quad M_{0} & =\frac{1}{2} q N(N-1)-\frac{k}{4}(N-1) .
\end{aligned}
$$

Here $\eta$ is the Dedekind function

$$
\eta(\tau)=\omega^{\frac{1}{24}} \prod_{n=1}^{\infty}\left(1-\omega^{n}\right)
$$


where $\omega=e^{2 \pi i \tau}$, and $\chi^{(k)}$ is the character for the affine $S U(2)$ Kac-Moody algebra at level $k$,

$$
\chi_{\lambda}^{(k)}(\tau)=\frac{\omega^{(\lambda+1)^{2} / 4(k+2)}}{\eta(\tau)^{3}} \sum_{n \in \mathbf{Z}}(\lambda+1+2 n(k+2)) \omega^{n(\lambda+1+(k+2) n)} .
$$

$\chi_{\lambda}^{(k)}$ can be decomposed into the spinon basis [38], in which the number of quasiparticle is explicit.

Annulus Let us next consider a state on an annulus. We first take a disk in the annulus which contains all the electrons, then we take a three-holed sphere whose holes are the two edges of the annulus and the boundary of the disk. By using duality transformations on CVOs [35], it can be expressed as [33],

$$
\Lambda\left(\begin{array}{c}
\beta_{2} \\
\beta_{1} \beta
\end{array}\right) \widetilde{\Phi}_{\text {disk }}\left(z_{1}, \cdots, z_{N}\right) .
$$

We will denote the vector space spanned by the expressions for edge excited states as $\Omega_{N}$.

We define the action of $M$ on $\Lambda$ by

$$
M \Lambda\left(\begin{array}{c}
j_{1} \\
j_{2} j_{3}
\end{array}\right)=\left(\Delta_{j_{1}}+\Delta_{j_{2}}-\Delta_{j_{3}}\right) \Lambda\left(\begin{array}{c}
j_{1} \\
j_{2} j_{3}
\end{array}\right)
$$

and introduce another operator $\bar{M}$ by

$$
\begin{aligned}
& \bar{M} \Phi\left(\begin{array}{c}
j_{1} \\
j_{2} j_{3}
\end{array}\right)=\left(\Delta_{j_{1}}+\Delta_{j_{2}}-\Delta_{j_{3}}\right) \Phi\left(\begin{array}{c}
j_{1} \\
j_{2} j_{3}
\end{array}\right) \\
& \bar{M} \Lambda\left(\begin{array}{c}
j_{1} \\
j_{2} j_{3}
\end{array}\right)=\left(\Delta_{j_{1}}+\Delta_{j_{2}}-\Delta_{j_{3}}\right) \Lambda\left(\begin{array}{c}
j_{1} \\
j_{2} j_{3}
\end{array}\right) .
\end{aligned}
$$

The pseudoenergy [9] is then given by $M_{E}=(M+\bar{M}) / 2$. We can also give the total charge operator by $Q=2(\bar{M}-M) /(2 q+k)$ for the expressions with positive $N$ and $Q=$ $2(M-\bar{M}) /(2 q+k)$ for the expressions with negative $N$. Then $\beta_{1}$ and $\beta_{2}$ satisfy

$$
\begin{aligned}
& \beta_{1}=\beta_{2} \quad \text { for } N: \text { even, } \\
& \beta_{1}=k-\beta_{2} \quad \text { for } N \text { : odd. }
\end{aligned}
$$

Moreover we must apply the selection rule for the charge sectors.

k:even First let us consider the case when $k$ is even. We denote the charge sectors for two edges as $m_{1}, m_{2}, \quad m_{1}, m_{2} \in \mathbf{Z}$. Since $k$ is even, if $m_{1}$ is from the even (odd) sector, $m_{2}$ is from the even (odd) sector and visa versa. They have a relation

$$
m_{1}=m_{2}+N q
$$

We can't determine $m_{1}$ and $m_{2}$ in this case. It is convenient to consider the grand canonical ensembles of electrons. In the Laughlin state, we gather all the charge sectors differing by integral charges into a single sector since integer charges are from the electrons. In spinonholon states, we must gather all the charge sectors differing by even integral charges into a single sector, since electrons are coupled by the degree of freedom from $S U(2)_{k}$. Thus 
we introduce the spaces $\Omega_{\text {edge }}^{\text {even }}=\bigoplus_{N \text { :even }} \Omega_{N}$ and $\Omega_{\text {edge }}^{\text {odd }}=\bigoplus_{N \text { :odd }} \Omega_{N}$ and the following characters:

$$
\begin{array}{r}
\chi_{r / q}^{\text {even }}(\tau, \zeta)=\frac{\mathrm{e}^{-\frac{\pi}{q} \frac{(\operatorname{Im} \zeta)^{2}}{\operatorname{Im} \tau}}}{\eta} \sum_{m \in Z_{\text {even }}} \mathrm{e}^{2 \pi i \tau \frac{(m q+r)^{2}}{2 q}+2 \pi i \zeta\left(m+\frac{r}{q}\right)}, \\
\chi_{r / q}^{\text {odd }}(\tau, \zeta)=\frac{\mathrm{e}^{-\frac{\pi}{q} \frac{(\operatorname{Im} \zeta)^{2}}{\operatorname{Im} \tau}}}{\eta} \sum_{m \in Z_{\text {odd }}} \mathrm{e}^{2 \pi i \tau \frac{(m q+r)^{2}}{2 q}+2 \pi i \zeta\left(m+\frac{r}{q}\right)}, \\
\chi_{(r+1 / 2) / q}^{\text {even }}(\tau, \zeta)=\frac{\mathrm{e}^{-\frac{\pi}{q} \frac{(\operatorname{Im} \zeta)^{2}}{\operatorname{Im} \tau}}}{\eta} \sum_{m \in Z_{\text {even }}} \mathrm{e}^{2 \pi i \tau \frac{(m q+r+1 / 2)^{2}}{2 q}+2 \pi i \zeta\left(m+\frac{r+1 / 2}{q}\right)} \\
\chi_{(r+1 / 2) / q}^{\text {odd }}(\tau, \zeta)=\frac{\mathrm{e}^{-\frac{\pi}{q} \frac{(\operatorname{Im} \zeta)^{2}}{\operatorname{Im} \tau}}}{\eta} \sum_{m \in Z_{\text {odd }}} \mathrm{e}^{2 \pi i \tau \frac{(m q+r+1 / 2)^{2}}{2 q}+2 \pi i \zeta\left(m+\frac{r+1 / 2}{q}\right)} .
\end{array}
$$

The prefactor will be necessary for the extended modular invariance conditions which will be explained in Sec.3.1. From these definitions, they satisfy

$$
\begin{gathered}
\chi_{r / q}^{\text {even }}=\chi_{-r / q}^{\text {even }}=\chi_{(2 q+r) / q}^{\text {even }}=\chi_{(q+r) / q}^{\text {odd }}, \\
\chi_{r / q}^{\text {odd }}=\chi_{-r / q}^{\text {odd }}=\chi_{(2 q+r) / q}^{\text {odd }}=\chi_{(q+r) / q}^{\text {even }} .
\end{gathered}
$$

From the couplings of $(2.12)$ and $(2.13)$, we see that the characters for the allowed excitations of chiral algebra become ( $a=$ even, odd):

$$
\begin{aligned}
& \chi_{j, r}^{a}=\chi_{j}^{(k)} \chi_{(r+1 / 2) / q}^{a}, \text { for } j: \text { odd } \\
& \chi_{j, r}^{a}=\chi_{j}^{(k)} \chi_{r / q}^{a} \text { for } j: \text { even. }
\end{aligned}
$$

Then, the grand-canonical partition function for odd $N$ is given by

$$
\begin{aligned}
Z^{\text {odd }}(\tau, \zeta) & =\operatorname{Tr}_{\Omega_{\text {edge }}^{\text {odd }}}\left(\mathrm{e}^{2 \pi i \tau\left(M_{E}-\frac{n_{b} c}{24}\right)+2 \pi i \zeta Q}\right) \\
& =\sum_{j=0}^{k} \sum_{r=0}^{q-1}\left[\chi_{j, r}^{\text {even }} \chi_{k-j, r}^{\text {odd }}+\chi_{j, r}^{\text {odd }} \chi_{k-j, r}^{\text {even }}\right]
\end{aligned}
$$

Also the grand-canonical partition function for even $N$ is given by

$$
\begin{aligned}
Z^{\text {even }}(\tau, \zeta) & =\operatorname{Tr}_{\Omega_{\text {edge }}^{\text {even }}}\left(\mathrm{e}^{2 \pi i \tau\left(M_{E}-\frac{n_{b} c}{24}\right)+2 \pi i \zeta Q}\right) \\
& =\sum_{j=0}^{k} \sum_{r=0}^{q-1}\left[\left(\chi_{j, r}^{\text {even }}\right)^{2}+\left(\chi_{j, r}^{\text {odd }}\right)^{2}\right]
\end{aligned}
$$

By introducing complex conjugate variables to distinguish the two edges, we see that the sum of these partition functions $Z^{\text {even }}+Z^{\text {odd }}$ is

$$
Z^{\text {ann }}(\tau, \zeta)=\sum_{j=0}^{k} \sum_{r=0}^{q-1}\left|\chi_{j, r}^{\text {even }}+\chi_{k-j, r}^{\text {odd }}\right|^{2}
$$

Thus we see that edge excitations of this system have $(k+1) q$ sectors. This number is the same as the number of the bulk excitations classified by their braiding property.

Except for the rational torus part, the annulus partition function eq.(2.44) becomes a $D$ type $\Gamma(2)$ modular invariants of $s \widehat{u(2)}$ in the recent classification by Gannon 13. When $k$ is a multiple of 4 , it is in the regime of $A D E$ classification of the $s \widehat{s(2)}$ modular invariants [32]. This is because the representation for the electron is the simple current which gives the corresponding automorphism of the fusion algebra to make $D$-type modular invariants. 
k:odd When $k$ is odd, $q$ is a half integer. Let $p$ be $2 q=2 s+k . p$ is an odd integer. We can rewrite the couplings (2.12)(2.13) as

$$
V_{j} e^{i \frac{m}{\sqrt{2 p}} \varphi}, \quad m=2 n+j \quad n \in \mathbf{N} .
$$

The charge sectors for the two edges $m_{1}, m_{2}, \quad m_{1}, m_{2} \in Z$ satisfy

$$
m_{1}=m_{2}+N q .
$$

When $N$ is even, $\beta_{1}=\beta_{2}$ as in (2.32). $m_{1}$ and $m_{2}$ are even or odd according to $\beta_{1}$ is even or odd. When $\beta_{1}$ is even, by putting $m_{1}=2 m_{1}^{\prime}, m_{2}=2 m_{2}^{\prime}, N=2 N^{\prime}$, 2.46) becomes

$$
m_{1}^{\prime}=m_{2}^{\prime}+N^{\prime} p
$$

When $\beta_{1}$ is odd, (2.46) reduces to (2.47) by putting $m_{1}-1=2 m_{1}^{\prime}, m_{2}-1=2 m_{2}^{\prime}$. Thus there are $p$ charge sectors.

When $N$ is odd, (2.46) can be rewritten as $\left(N=2 N^{\prime}-1\right)$

$$
m_{1}=m_{2}+p+2 N^{\prime} p .
$$

Since $\beta_{1}=k-\beta_{2}$ as in (2.32) and $k$ is odd, $\beta_{1}\left(m_{1}\right)$ and $\beta_{2}\left(m_{2}\right)$ have opposite parities. When $\beta_{1}$ is even and $\beta_{2}$ is odd, we can put $m_{1}=2 m_{1}^{\prime}, m_{2}=2 m_{2}^{\prime}-p$. Then (2.48) again reduces to the form (2.47). The case that $\beta_{1}$ is odd is similarly worked out. Thus there are also $p$ charge sectors.

Accordingly we introduce the characters:

$$
\begin{aligned}
\chi_{r / p}(\tau, \zeta) & =\chi_{r / p}^{\mathrm{even}}(\tau, \zeta)+\chi_{r / p}^{\mathrm{odd}}(\tau, \zeta) \\
& =\frac{\mathrm{e}^{-\frac{\pi}{p} \frac{(\operatorname{Im} \zeta)^{2}}{\operatorname{Im} \tau}}}{\eta} \sum_{m \in Z} \mathrm{e}^{2 \pi i \tau \frac{(m p+r)^{2}}{2 p}+2 \pi i \zeta\left(m+\frac{r}{p}\right)} .
\end{aligned}
$$

From the consideration above, it is convenient to introduce the following characters:

$$
\begin{aligned}
& \chi_{j, r}=\chi_{j}^{(k)} \chi_{(2 r+p) / 2 p}^{a}, \text { for } j \text { : odd } \\
& \chi_{j, r}=\chi_{j}^{(k)} \chi_{2 r / 2 p}^{a} \text { for } j \text { : even. }
\end{aligned}
$$

Then the grand partition function for even $N$ becomes

$$
\begin{aligned}
Z^{\text {even }}(\tau, \zeta) & =\operatorname{Tr}_{\Omega_{\text {edge }}^{\text {even }}}\left(\mathrm{e}^{2 \pi i \tau\left(M_{E}-\frac{n_{b} c}{24}\right)+2 \pi i \zeta Q}\right) \\
& =\sum_{j \text { :even }} \sum_{r=0}^{p-1}\left[\left(\chi_{j, r}\right)^{2}+\left(\chi_{k-j, r}\right)^{2}\right] .
\end{aligned}
$$

Also the grand-canonical partition function for odd $N$ becomes

$$
\begin{aligned}
Z^{\text {odd }}(\tau, \zeta) & =\operatorname{Tr}_{\Omega_{\text {edge }}^{\text {odd }}}\left(\mathrm{e}^{2 \pi i \tau\left(M_{E}-\frac{n_{b} c}{24}\right)+2 \pi i \zeta Q}\right) \\
& =\sum_{j \text { :even }} \sum_{r=0}^{p-1} 2 \chi_{j, r} \chi_{k-j, r} .
\end{aligned}
$$

Then the grand partition function $Z^{\text {even }}+Z^{\text {odd }}$ becomes

$$
Z^{\text {ann }}(\tau, \zeta)=\sum_{r=0}^{p-1} \sum_{j: \text { even }}\left|\chi_{j, r}+\chi_{k-j, r}\right|^{2} .
$$

Thus there are $(k+1) q$ sectors of edge excitations as in the even $k$ case. However the Hilbert space of edge excitations is decomposed in a different way. 
Multiple Boundaries We can also deduce the grand partition function for the FQH state on multiply connected region.

k: even Next let us consider a spinon-holon FQH state on a region with $n_{b}$ boundaries. In this case, we need at least $n_{b}-1$ CVOs to express the $n_{b}$ boundaries. As in the case of an annulus, we end up with an expression,

$$
\Lambda\left(\begin{array}{c}
\beta_{2} \\
\beta_{1} \alpha_{1}
\end{array}\right) \Lambda\left(\begin{array}{c}
\beta_{3} \\
\alpha_{1} \alpha_{2}
\end{array}\right) \cdots \Lambda\left(\begin{array}{c}
\beta_{n_{b}} \\
\alpha_{n_{b}-2} \beta
\end{array}\right) \widetilde{\Phi}_{\text {disk }}\left(z_{1}, \cdots, z_{N}\right)
$$

Let us denote the partition function of edge excitations with $\beta=(j, \lambda, a)$ where $j=$ $1, \cdots, k, \quad \lambda=0, \cdots, q-1, a=$ even, odd as $Z_{i, \lambda}^{a,\left(n_{b}\right)}$. By summing over all the sectors of $\beta_{n_{b}}$, we find the equation satisfied by $Z_{i, \lambda}^{a,\left(n_{b}\right)}$ :

$$
Z_{i, s}^{a_{1},\left(n_{b}\right)}=\sum_{j k} \sum_{a_{2}+a_{3}=a_{1}} \sum_{\lambda=0}^{q-1} N_{i j}^{k} \chi_{j, \lambda}^{a_{2}} Z_{k, s+\lambda}^{a_{3},\left(n_{b}-1\right)}
$$

Here $N_{i j}^{k}$ is the fusion rules of $S U(2)_{k}$ WZW model in (2.2) and $Z^{\left(n_{b}-1\right)}$ is the partition function for the remaining $\left(n_{b}-1\right)$ boundaries. We can diagonalize this equation by using the Verlinde formula 33]

$$
\begin{aligned}
N_{j k}^{i} & =\sum_{n} S_{j}^{n} \lambda_{k}^{(n)} S_{n}^{\dagger i}, \\
\lambda_{k}^{(n)} & =S_{k}^{n} / S_{0}^{n},
\end{aligned}
$$

where $S_{i}^{j}$ is the matrix element of modular transformation on the Virasoro characters. The modular behavior of the characters $\chi_{l}^{(k)}$ are given by 37, 36

$$
S_{l n}^{(k)}=\left(\frac{2}{k+2}\right)^{1 / 2} \sin \frac{(l+1)(n+1)}{k+2} \pi .
$$

By using the modular matrix element, we obtain the partition functions as :

$$
Z_{l, s}^{a,\left(n_{b}\right)}=\sum_{n=0}^{k}\left(\frac{2}{k+2}\right)^{1 / 2} \sin \left(\frac{(l+1)(n+1)}{k+2} \pi\right) \Xi_{n, s}^{a,\left(n_{b}\right)},
$$

where $\Xi_{n . s}^{a,\left(n_{b}\right)}$ is

$$
\begin{gathered}
\Xi_{n, s}^{a,\left(n_{b}\right)}=\left(\frac{2}{k+2}\right)^{1 / 2} \sin \frac{n+1}{k+2} \pi \sum_{p_{2}, \cdots, p_{n}} \sum_{a_{2}, \cdots, a_{n_{b}}} \xi_{n, p_{1}}^{a_{1}} \cdots \xi_{n, p_{n_{b}}}^{a_{n_{b}}}, \\
p_{1} \equiv s+\sum_{l=2}^{n_{b}} p_{l}, \quad(\bmod 2 q), \quad a_{1} \equiv a+\sum_{l=2}^{n_{b}} a_{l}, \\
\xi_{n, p}^{a}=\sum_{j=0}^{k}\left[\frac{\sin \frac{(n+1)(j+1)}{k+2} \pi}{\sin \frac{n+1}{k+2} \pi}\right] \chi_{j, \lambda}^{a} .
\end{gathered}
$$

In particular, the grand-canonical partition function $Z_{0,0}^{\text {even }}+Z_{k, 0}^{\text {odd }}$ is

$$
Z=\left(\frac{2}{k+2}\right)^{1 / 2} \sum_{n=0}^{k}\left[\sin \left(\frac{n+1}{k+2} \pi\right)\left(\Xi_{n, s}^{\text {even },\left(n_{b}\right)}+(-1)^{n+1} \Xi_{n, s}^{\text {odd },\left(n_{b}\right)}\right)\right] .
$$


k:odd In this case, we have $p$ different sectors for the charge sector and no distinction between even and odd sectors. Thus we get

$$
Z_{i, s}^{\left(n_{b}\right)}=\sum_{j k} \sum_{\lambda=0}^{2 p-1} N_{i j}^{k} \chi_{j, \lambda} Z_{k, s+\lambda}^{\left(n_{b}-1\right)}
$$

Accordingly we get

$$
Z_{l, s}^{\left(n_{b}\right)}=\sum_{n=0}^{k}\left(\frac{2}{k+2}\right)^{1 / 2} \sin \left(\frac{(l+1)(n+1)}{k+2} \pi\right) \Xi_{n, s}^{\left(n_{b}\right)},
$$

where $\Xi_{n . s}^{\left(n_{b}\right)}$ is

$$
\begin{gathered}
\Xi_{n, s}^{\left(n_{b}\right)}=\left(\frac{2}{k+2}\right)^{1 / 2} \sin \frac{n+1}{k+2} \pi \sum_{p_{2}, \cdots, p_{n}} \xi_{n, p_{1}} \cdots \xi_{n, p_{n_{b}}}, \\
p_{1} \equiv s+\sum_{l=2}^{n_{b}} p_{l}, \quad(\bmod p), \\
\xi_{n, \lambda}=\sum_{j=0}^{k}\left[\frac{\sin \frac{(n+1)(j+1)}{k+2} \pi}{\sin \frac{n+1}{k+2} \pi}\right] \chi_{j, \lambda} .
\end{gathered}
$$

In particular, the grand-canonical partition function $Z_{0,0}+Z_{k, 0}$ is

$$
Z=\left(\frac{2}{k+2}\right)^{1 / 2} \sum_{n=0}^{k}\left[\sin \left(\frac{n+1}{k+2} \pi\right)\left(\Xi_{n, s}^{\left(n_{b}\right)}+(-1)^{n+1} \Xi_{n, s}^{\left(n_{b}\right)}\right)\right] .
$$

The partition function for the Halperin state is the $k=1$ case.

\section{Modular Invariance and the Topological Order}

The annulus partition functions for paired states (Pfaffian, Haldane-Rezayi, 331) were derived in [9], but the modular properties of the extended characters have not been worked out. For the Haldane-Rezayi state, the modular invariant partition function was discussed in [29], but only for the internal degrees of freedom given by the $c=-2$ scalar fermion. In this section, we investigate the full modular behaviors of the characters including all the degrees of freedom and verify the modular invariance of annulus partition functions for the Pfaffian, 331 and spinon-holon states. This enable us to extend the observation of Cappelli and Zemba on the relation between topological order and modular properties for the characters to these states. For the Haldane-Rezayi state, we find a pathologic behavior due to the coupling between the internal degrees of freedom and the charge degrees of freedom.

\subsection{Modular Invariance Conditions}

In Ref. 10] Cappelli and Zemba gave the following extended modular invariance conditions for the annulus partition function of a FQH state:

$$
\begin{aligned}
(S): & Z\left(-\frac{1}{\tau},-\frac{\zeta}{\tau}\right) & =Z(\tau, \zeta) . \\
\left(T^{2}\right): & Z(\tau+2, \zeta) & =Z(\tau, \zeta) . \\
(U): & Z(\tau, \zeta+1) & =Z(\tau, \zeta) . \\
(V): & Z(\tau, \zeta+\tau) & =Z(\tau, \zeta) .
\end{aligned}
$$


The physical meanings of these conditions are as follows. The edge theory is equivalent to Chern-Simons theory on the 3-manifold, Annulus $\times S^{1}\left(S^{1}\right.$ is the Euclidean time) [23]. The annulus partition function is the amplitude in Chern-Simons theory. Then it must be invariant under the modular transformation on the modular parameter of Euclidean spacetime or space-space torus. This gives the condition $(S)$.

The condition $\left(T^{2}\right)$ means that the FQH state is consist of electrons and the physical excitations must have integer or half integer spin. Also the total charge of the state must be integer, which gives the condition $(U)$.

The final condition $(V)$ is first considered by Laughlin [2]. It is a consequence of gauge invariance : the addition of a quantum of flux through the center of the annulus is a symmetry of the gauge-invariant Hamiltonian but causes a flow of all the quantum states among themselves (spectral flow). The spectral flow can be simulated by requiring the invariance of the partition functions under a shift of the electric potential by $\zeta \rightarrow \zeta+\tau$. This shift causes the transport of a fractional charge between the two edges and thus determine the Hall conductivity.

Among the transformations $S, T^{2}, U$, and $V$, the transformations $S T^{2} S$ and $S$ generate the subgroup $\Gamma(2)$ of the modular group $\Gamma=S L(2, \mathbf{Z}) / \mathbf{Z}_{2}$ of rational transformations $\tau \rightarrow$ $(a \tau+b) /(c \tau+d), a, b, c, d \in \mathbf{Z}$, which are subjected to the conditions $(a, d)$ odd and $(b, c)$ even. Thus, $Z$ is invariant under the modular subgroup $\Gamma(2)$ *

\subsection{Modular Behavior of U(1) Characters}

As we saw in Sec.2, the coupling of chiral boson to internal degrees of freedom are determined by the single-valuedness and nonsingularity of wave function in electron's coordinates. When we consider the grand-canonical ensemble of electrons, we end up with the characters :

$$
\chi_{r / q}^{\text {even }}(\tau, \zeta), \quad \chi_{r / q}^{\text {odd }}(\tau, \zeta), \quad \chi_{(r+1 / 2) / q}^{\text {even }}(\tau, \zeta), \quad \chi_{(r+1 / 2) / q}^{\text {odd }}(\tau, \zeta) .
$$

Let us determine the modular behavior of these characters. To that end, we recall the character for $q \rightarrow 4 q$ :

$$
\chi_{r / 4 q}(\tau, \zeta)=\frac{\mathrm{e}^{-\frac{\pi}{4 q} \frac{(\operatorname{Im} \zeta)^{2}}{\operatorname{Im} \tau}}}{\eta} \sum_{m \in Z} \mathrm{e}^{2 \pi i \tau \frac{(4 q m+r)^{2}}{8 q}+2 \pi i \zeta\left(m+\frac{r}{4 q}\right)} .
$$

By comparing (2.342.37) with (3.5), we see that these characters are related as

$$
\begin{aligned}
\chi_{r / q}^{\text {even }}(\tau, \zeta) & =\chi_{2 r / 4 q}(\tau, 2 \zeta), \\
\chi_{r / q}^{\text {odd }}(\tau, \zeta) & =\chi_{(2 r+2 q) / 4 q}(\tau, 2 \zeta), \\
\chi_{(r+1 / 2) / q}^{\text {even }}(\tau, \zeta) & =\chi_{(2 r+1) / 4 q}(\tau, 2 \zeta), \\
\chi_{(r+1 / 2) / q}^{\text {odd }}(\tau, \zeta) & =\chi_{(2 r+2 q+1) / 4 q}(\tau, 2 \zeta) .
\end{aligned}
$$

Let us now examine the transformation properties of the characters. First, the transformations $T^{2}, S, U, V$ of the character $\chi_{\lambda / q}$ are :

$$
S: \chi_{\lambda / q}\left(-\frac{1}{\tau},-\frac{\zeta}{\tau}\right)=\frac{\mathrm{e}^{i \frac{\pi}{q} \operatorname{Re} \frac{\zeta^{2}}{\tau}}}{\sqrt{q}} \sum_{\lambda^{\prime}=0}^{q-1} \mathrm{e}^{2 \pi i \frac{\lambda \lambda^{\prime}}{q}} \chi_{\lambda^{\prime} / q}(\tau, \zeta)
$$

${ }^{*} S$ and $T^{2}$ actually generate a slightly larger subgroup than $\Gamma(2)$. See the appendix of Ref. 10] 


$$
\begin{aligned}
T^{2}: \chi_{\lambda / q}(\tau+2, \zeta) & =\mathrm{e}^{2 \pi i\left(\frac{\lambda^{2}}{q}-\frac{1}{12}\right)} \chi_{\lambda / q}(\tau, \zeta) \\
U: \chi_{\lambda / q}(\tau, \zeta+1) & =\mathrm{e}^{\frac{2 \pi i \lambda}{q}} \chi_{\lambda / q}(\tau, \zeta) \\
V: \chi_{\lambda / q}(\tau, \zeta+\tau) & =\mathrm{e}^{-\frac{2 \pi i}{q}\left(\operatorname{Re} \zeta+\operatorname{Re} \frac{\tau}{2}\right)} \chi_{(\lambda+1) / q}(\tau, \zeta)
\end{aligned}
$$

These transformations show that the characters $\chi_{\lambda} / q$ carry a unitary representation of the modular group $\Gamma(2)$, which is projective for $\zeta \neq 0$ (the composition law is verified up to a phase). Actually, the prefactor is necessary to transform $\chi_{\lambda / q}$ by phase under the spectral flow $V$ [10]. The same prefactor also appears in the quantization of the Chern-Simons theory on the space torus, in the measure for the inner product of the wave functions [23].

From (3.6 3.9), the modular behaviors of $\chi_{r / q}^{\text {even }}, \chi_{r / q}^{\text {odd }}, \chi_{(r+1 / 2) / q}^{\text {even }}, \chi_{(r+1 / 2) / q}^{\text {odd }}$ under $T^{2}, U, V$ are the same with $(3.11)(3.12)(3.13)$. Also the modular behavior under $S$ is determined to be

$$
\begin{aligned}
& \chi_{r / q}^{\text {even }} \rightarrow \frac{\mathrm{e}^{i \frac{\pi}{q} \operatorname{Re} \frac{\zeta^{2}}{\tau}}}{2 \sqrt{q}} \sum_{s=0}^{q-1}\left\{\mathrm{e}^{\frac{2 \pi i r s}{q}}\left(\chi_{s / q}^{\text {even }}+\chi_{s / q}^{\text {odd }}\right)+\mathrm{e}^{\frac{2 \pi i r\left(s+\frac{1}{2}\right)}{q}}\left(\chi_{(s+1 / 2) / q}^{\text {even }}+\chi_{(s+1 / 2) / q}^{\text {odd }}\right)\right\} \\
& \chi_{r / q}^{\text {odd }} \rightarrow \frac{\mathrm{e}^{i \frac{\pi}{q} \operatorname{Re} \frac{\zeta^{2}}{\tau}}}{2 \sqrt{q}} \sum_{s=0}^{q-1}\left\{\mathrm{e}^{\frac{2 \pi i r s}{q}}\left(\chi_{s / q}^{\text {even }}+\chi_{s / q}^{\text {odd }}\right)-\mathrm{e}^{\frac{2 \pi i r\left(s+\frac{1}{2}\right)}{q}}\left(\chi_{(s+1 / 2) / q}^{\text {even }}+\chi_{(s+1 / 2) / q}^{\text {odd }}\right)\right\} \\
& \chi_{(r+1 / 2) / q}^{\text {even }} \rightarrow \frac{\mathrm{e}^{i \frac{\pi}{q} \operatorname{Re} \frac{\zeta^{2}}{\tau}}}{2 \sqrt{q}} \sum_{s=0}^{q-1}\left\{\mathrm{e}^{\frac{2 \pi i\left(r+\frac{1}{2}\right) s}{q}}\left(\chi_{s / q}^{\text {even }}-\chi_{s / q}^{\text {odd }}\right)+\mathrm{e}^{\frac{2 \pi i\left(r+\frac{1}{2}\right)\left(s+\frac{1}{2}\right)}{q}}\left(\chi_{(s+1 / 2) / q}^{\text {even }}-\chi_{(s+1 / 2) / q}^{\text {odd }}\right)\right\} \\
& \chi_{(r+1 / 2) / q}^{\text {even }} \rightarrow \frac{\mathrm{e}^{i \frac{\pi}{q} \operatorname{Re} \frac{\zeta^{2}}{\tau}}}{2 \sqrt{q}} \sum_{s=0}^{q-1}\left\{\mathrm{e}^{\frac{2 \pi i\left(r+\frac{1}{2}\right) s}{q}}\left(\chi_{s / q}^{\text {even }}-\chi_{s / q}^{\text {odd }}\right)-\mathrm{e}^{\frac{2 \pi i\left(r+\frac{1}{2}\right)\left(s+\frac{1}{2}\right)}{q}}\left(\chi_{(s+1 / 2) / q}^{\text {even }}-\chi_{(s+1 / 2) / q}^{\text {odd }}\right)\right\} .
\end{aligned}
$$

\subsection{Spinon-Holon States}

Let us examine the modular behavior of characters for spinon-holon states.

k: even From (2.44), there are $(k+1) q$ sectors:

$$
\chi_{j, r}^{\mathrm{sph}}=\chi_{j, r}^{\mathrm{even}}+\chi_{k-j, r}^{\mathrm{odd}}, \quad j=0, \cdots, k, \quad r=0, \cdots, q-1 .
$$

Combining the modular behaviors in (2.59) and (3.14 3.17), we get the modular behavior of $\chi_{j, r}^{\mathrm{sph}}$. When $j$ is even, we have

$$
\begin{aligned}
& \chi_{j, r}^{\text {even }} \rightarrow \frac{\mathrm{e}^{i \frac{\pi}{q} \operatorname{Re} \frac{\zeta^{2}}{\tau}}}{2 \sqrt{q}} \sum_{n=0}^{k} \sum_{s=0}^{q-1} S_{j n}^{(k)}\left\{\mathrm{e}^{\frac{2 \pi i r s}{q}}\left(\chi_{n, s}^{\text {even }}+\chi_{n, s}^{\text {odd }}\right)+\mathrm{e}^{\frac{2 \pi i r\left(s+\frac{1}{2}\right)}{q}}\left(\chi_{n, s+1 / 2}^{\text {even }}+\chi_{n, s+1 / 2}^{\text {odd }}\right)\right\} \\
&(3.19) \\
& \chi_{j, r}^{\text {odd }} \rightarrow \frac{\mathrm{e}^{i \frac{\pi}{q} \operatorname{Re} \frac{\zeta^{2}}{\tau}}}{2 \sqrt{q}} \sum_{n=0}^{k} \sum_{s=0}^{q-1} S_{j n}^{(k)}\left\{\mathrm{e}^{\frac{2 \pi i r s}{q}}\left(\chi_{n, s}^{\text {even }}+\chi_{n, s}^{\text {odd }}\right)-\mathrm{e}^{\frac{2 \pi i r\left(s+\frac{1}{2}\right)}{q}}\left(\chi_{n, s+1 / 2}^{\text {even }}+\chi_{n, s+1 / 2}^{\text {odd }}\right)\right\},
\end{aligned}
$$


When $j$ is odd, we have

$$
\begin{aligned}
& \chi_{j, r}^{\text {even }} \rightarrow \frac{\mathrm{e}^{i \frac{\pi}{q} \operatorname{Re} \frac{\zeta^{2}}{\tau}}}{2 \sqrt{q}} \sum_{n=0}^{k} \sum_{s=0}^{q-1} S_{j n}^{(k)}\left\{\mathrm{e}^{\frac{2 \pi i\left(r+\frac{1}{2}\right) s}{q}}\left(\chi_{n, s}^{\text {even }}-\chi_{n, s}^{\text {odd }}\right)+\mathrm{e}^{\frac{2 \pi i\left(r+\frac{1}{2}\right)\left(s+\frac{1}{2}\right)}{q}}\left(\chi_{n, s+1 / 2}^{\text {even }}-\chi_{n, s+1 / 2}^{\text {odd }}\right)\right\}, \\
& \chi_{j, r}^{\text {odd }} \rightarrow \frac{\mathrm{e}^{i \frac{\pi}{q} \operatorname{Re} \frac{\zeta^{2}}{\tau}}}{2 \sqrt{q}} \sum_{n=0}^{k} \sum_{s=0}^{q-1} S_{j n}^{(k)}\left\{\mathrm{e}^{\frac{2 \pi i\left(r+\frac{1}{2}\right) s}{q}}\left(\chi_{n, s}^{\text {even }}-\chi_{n, s}^{\text {odd }}\right)-\mathrm{e}^{\frac{2 \pi i r\left(s+\frac{1}{2}\right)}{q}}\left(\chi_{n, s+1 / 2}^{\text {even }}-\chi_{n, s+1 / 2}^{\text {odd }}\right)\right\} .
\end{aligned}
$$

By noticing

$$
S_{(k-l) n}^{(k)}=(-1)^{n} S_{l n}^{(k)}
$$

we see that the modular behavior of $\chi_{j, r}^{\mathrm{sph}}$ is given by ,

$\widetilde{S}: \chi_{j, r}^{\mathrm{sph}} \rightarrow \frac{\mathrm{e}^{i \frac{\pi}{q} \operatorname{Re} \frac{\zeta^{2}}{\tau}}}{\sqrt{q}}\left\{\sum_{n: \text { even }} \sum_{s=0}^{q-1} S_{j n}^{(k)} \mathrm{e}^{\frac{2 \pi i r s}{q}} \chi_{n, s}^{\mathrm{sph}}+\sum_{n: \text { odd }} \sum_{s=0}^{q-1} S_{j n}^{(k)} \mathrm{e}^{\frac{2 \pi i r\left(s+\frac{1}{2}\right)}{q}} \chi_{n, s}^{\mathrm{sph}}\right\}$, for $j$ even, (3.24)
$\widetilde{S}: \chi_{j, r}^{\mathrm{sph}} \rightarrow \frac{\mathrm{e}^{i \frac{\pi}{q} \operatorname{Re} \frac{\zeta^{2}}{\tau}}}{\sqrt{q}}\left\{\sum_{n: \text { even }} \sum_{s=0}^{q-1} S_{j n}^{(k)} \mathrm{e}^{\frac{2 \pi i\left(r+\frac{1}{2}\right) s}{q}} \chi_{n, s}^{\mathrm{sph}}+\sum_{n: \text { odd }} \sum_{s=0}^{q-1} S_{j n}^{(k)} \mathrm{e}^{\frac{2 \pi i\left(r+\frac{1}{2}\right)\left(s+\frac{1}{2}\right)}{q}} \chi_{n, s}^{\mathrm{sph}}\right\}$, for $j$ odd.

Thus $\chi_{j, r}^{\text {sph }}$ for $j$ :even, odd form an irreducible representation of the modular group. It is readily seen that the annulus partition function (2.44) is invariant under $(S)$. The conditions $\left(T^{2}\right),(U)$, and $(V)$ are verified similarly. We also see that $\widetilde{S}$ reproduces the correct fusion rules of the representations in this model through the Verlinde formula (3.60).

k:odd (2.54) can be rewritten as

$$
Z^{\mathrm{ann}}(\tau, \zeta)=\frac{1}{2} \sum_{r=0}^{p-1} \sum_{j=0}^{k}\left|\chi_{j, r}+\chi_{k-j, r}\right|^{2}
$$

Thus the sectors of edge excitations are

$$
\chi_{j, r}^{\mathrm{sph}}=\chi_{j, r}+\chi_{k-j, r}, \quad j=0, \cdots, k, \quad r=0, \cdots, p-1 .
$$

In this case, $\chi_{j, r}$ transforms as

$$
\begin{aligned}
& \chi_{j, r} \rightarrow \frac{\mathrm{e}^{i \frac{\pi}{q} \operatorname{Re} \frac{\zeta^{2}}{\tau}}}{2 \sqrt{q}} \sum_{s=0}^{2 p-1} \sum_{n=0}^{k} \mathrm{e}^{\frac{2 \pi i r s}{p}} S_{j n}^{(k)} \chi_{n, s / 2} \quad \text { for } j: \text { even } \\
& \chi_{j, r} \rightarrow \frac{\mathrm{e}^{i \frac{\pi}{q} \operatorname{Re} \frac{\zeta^{2}}{\tau}}}{2 \sqrt{q}} \sum_{s=0}^{2 p-1} \sum_{n=0}^{k} \mathrm{e}^{\frac{2 \pi i r s}{p}} \mathrm{e}^{i \pi s} S_{j n}^{(k)} \chi_{n, s / 2} \quad \text { for } j: \text { odd }
\end{aligned}
$$

Therefore, from (3.23), $\chi_{j, r}^{\mathrm{sph}}$ transforms as

$$
\chi_{j, r}^{\mathrm{sph}} \rightarrow \frac{\mathrm{e}^{i \frac{\pi}{q} \operatorname{Re} \frac{\zeta^{2}}{\tau}}}{\sqrt{q}} \sum_{s=0}^{p-1} \sum_{n=0}^{k} \mathrm{e}^{\frac{2 \pi i r s}{p}} S_{j n}^{(k)} \chi_{n, s}^{\mathrm{sph}} .
$$


We see that the state realizes a different representation from the even $k$ case (3.25). Obviously the annulus partition function for odd $k(3.26)$ is invariant under this transformation. The conditions $\left(T^{2}\right),(U)$, and $(V)$ are verified similarly. The fusion rules are also reproduced from the Verlinde formula.

The degeneracy of the state on a genus $g$ Riemann surface is $(k+1)^{g} q^{g}$ for both even and odd $k$. However the structures of the topological order are different as we see from (3.25) and (3.30).

\subsection{Paired States}

In this section, we examine the modular properties of characters for paired states. We first present the analysis for the Pfaffian state [14 in detail.

\subsubsection{The Pfaffian state}

The annulus partition function for the Pfaffian state at $\nu=\frac{1}{q}$ is given by 9

$$
\begin{aligned}
Z_{\mathrm{Pf}}^{\mathrm{ann}} & =\sum_{r=0}^{q-1}\left\{\left|\chi_{1, r}^{\mathrm{even}}+\chi_{\psi, r}^{\mathrm{odd}}\right|^{2}+\left|\chi_{\psi, r}^{\mathrm{even}}+\chi_{1, r}^{\mathrm{odd}}\right|^{2}+\left|\chi_{\sigma, r}^{\mathrm{even}}+\chi_{\sigma, r}^{\mathrm{odd}}\right|^{2}\right\} \\
\chi_{i, r}^{a} & =\chi_{i}^{\mathrm{MW}} \chi_{r / q}^{a}, \quad i=1, \psi, \\
\chi_{\sigma, r+\frac{1}{2}}^{a} & =\chi_{\sigma}^{\mathrm{MW}} \chi_{(r+1 / 2) / q}^{a},
\end{aligned}
$$

where $\chi_{1}^{\mathrm{MW}}, \chi_{\psi}^{\mathrm{MW}}, \chi_{\sigma}^{\mathrm{MW}}$ are the characters for the Ising model:

$$
\begin{aligned}
\chi_{1}^{\mathrm{MW}}(\tau) & =\frac{1}{2} \omega^{-\frac{1}{48}}\left(\prod_{0}^{\infty}\left(1+\omega^{n+\frac{1}{2}}\right)+\prod_{0}^{\infty}\left(1-\omega^{n+\frac{1}{2}}\right)\right) \\
& =\frac{1}{2} \sqrt{\frac{\theta_{3}(\tau)}{\eta(\tau)}}+\frac{1}{2} \sqrt{\frac{\theta_{4}(\tau)}{\eta(\tau)}}, \\
\chi_{\sigma}^{\mathrm{MW}}(\tau) & =\omega^{\frac{1}{24}} \prod_{1}^{\infty}\left(1+\omega^{n}\right) \\
& =\sqrt{\frac{\theta_{2}(\tau)}{2 \eta(\tau)}}, \\
\chi_{\psi}^{\mathrm{MW}}(\tau) & =\frac{1}{2} \omega^{-\frac{1}{48}}\left(\prod_{0}^{\infty}\left(1+\omega^{n+\frac{1}{2}}\right)-\prod_{0}^{\infty}\left(1-\omega^{n+\frac{1}{2}}\right)\right) \\
& =\frac{1}{2} \sqrt{\frac{\theta_{3}(\tau)}{\eta(\tau)}}-\frac{1}{2} \sqrt{\frac{\theta_{4}(\tau)}{\eta(\tau)}} .
\end{aligned}
$$

Here $\theta_{2}, \theta_{3}, \theta_{4}$ are Jacobi theta functions. The matrix elements of modular transformation for the Virasoro characters $\chi_{1}^{\mathrm{MW}}, \chi_{\sigma}^{\mathrm{MW}}, \chi_{\psi}^{\mathrm{MW}}$ follow from the transformation property of the Jacobi $\theta$ functions as

$$
S_{\text {Ising }}=\frac{1}{2}\left(\begin{array}{ccc}
1 & \sqrt{2} & 1 \\
\sqrt{2} & 0 & -\sqrt{2} \\
1 & -\sqrt{2} & 1
\end{array}\right)
$$


From (3.31), we see that there are $3 q$ sectors in the edge excitations of the Pfaffian state:

$$
\begin{aligned}
\chi_{1, r}^{\mathrm{Pf}} & =\chi_{1, r}^{\text {even }}+\chi_{\psi, r}^{\text {odd }} \\
\chi_{\psi, r}^{\mathrm{Pf}} & =\chi_{\psi, r}^{\text {even }}+\chi_{1, r}^{\text {odd }} \\
\chi_{\sigma, r+1 / 2}^{\mathrm{Pf}} & =\chi_{\sigma, r+1 / 2}^{\text {even }}+\chi_{\sigma, r+1 / 2}^{\text {odd }}
\end{aligned}
$$

From (3.40) and (3.17), we get the modular behaviors

$$
\begin{aligned}
& \chi_{1, r}^{\text {even }} \rightarrow \frac{\mathrm{e}^{i \frac{\pi}{q} \operatorname{Re} \frac{\zeta^{2}}{\tau}}}{2 \sqrt{q}} \sum_{s=0}^{q-1}\left\{\frac{1}{2}\left(\mathrm{e}^{\frac{2 \pi i r s}{q}}\left(\chi_{1, s}^{\text {even }}+\chi_{1, s}^{\text {odd }}\right)+\mathrm{e}^{\frac{2 \pi i r\left(s+\frac{1}{2}\right)}{q}}\left(\chi_{1, s+1 / 2}^{\text {even }}+\chi_{1, s+1 / 2}^{\text {odd }}\right)\right)\right. \\
& +\frac{\sqrt{2}}{2}\left(\mathrm{e}^{\frac{2 \pi i r s}{q}}\left(\chi_{\sigma, s}^{\mathrm{even}}+\chi_{\sigma, s}^{\mathrm{odd}}\right)+\mathrm{e}^{\frac{2 \pi i r\left(s+\frac{1}{2}\right)}{q}}\left(\chi_{\sigma, s+1 / 2}^{\mathrm{even}}+\chi_{\sigma, s+1 / 2}^{\mathrm{odd}}\right)\right) \\
& \left.+\frac{1}{2}\left(\mathrm{e}^{\frac{2 \pi i r s}{q}}\left(\chi_{\psi, s}^{\text {even }}+\chi_{\psi, s}^{\text {odd }}\right)+\mathrm{e}^{\frac{2 \pi i r\left(s+\frac{1}{2}\right)}{q}}\left(\chi_{\psi, s+1 / 2}^{\text {even }}+\chi_{\psi, s+1 / 2}^{\text {odd }}\right)\right)\right\}, \\
& \chi_{1, r}^{\text {odd }} \rightarrow \frac{\mathrm{e}^{i \frac{\pi}{q} \operatorname{Re} \frac{\zeta^{2}}{\tau}}}{2 \sqrt{q}} \sum_{s=0}^{q-1}\left\{\frac{1}{2}\left(\mathrm{e}^{\frac{2 \pi i r s}{q}}\left(\chi_{1, s}^{\text {even }}+\chi_{1, s}^{\text {odd }}\right)-\mathrm{e}^{\frac{2 \pi i r\left(s+\frac{1}{2}\right)}{q}}\left(\chi_{1, s+1 / 2}^{\text {even }}+\chi_{1, s+1 / 2}^{\text {odd }}\right)\right)\right. \\
& +\frac{\sqrt{2}}{2}\left(\mathrm{e}^{\frac{2 \pi i r s}{q}}\left(\chi_{\sigma, s}^{\mathrm{even}}+\chi_{\sigma, s}^{\mathrm{odd}}\right)-\mathrm{e}^{\frac{2 \pi i r\left(s+\frac{1}{2}\right)}{q}}\left(\chi_{\sigma, s+1 / 2}^{\mathrm{even}}+\chi_{\sigma, s+1 / 2}^{\mathrm{odd}}\right)\right) \\
& \left.+\frac{1}{2}\left(\mathrm{e}^{\frac{2 \pi i r s}{q}}\left(\chi_{\psi, s}^{\text {even }}+\chi_{\psi, s}^{\text {odd }}\right)-\mathrm{e}^{\frac{2 \pi i r\left(s+\frac{1}{2}\right)}{q}}\left(\chi_{\psi, s+1 / 2}^{\text {even }}+\chi_{\psi, s+1 / 2}^{\text {odd }}\right)\right)\right\}, \\
& \chi_{1, r}^{\text {even }} \rightarrow \frac{\mathrm{e}^{i \frac{\pi}{q} \operatorname{Re} \frac{\zeta^{2}}{\tau}}}{2 \sqrt{q}} \sum_{s=0}^{q-1}\left\{\frac{1}{2}\left(\mathrm{e}^{\frac{2 \pi i r s}{q}}\left(\chi_{1, s}^{\text {even }}+\chi_{1, s}^{\text {odd }}\right)+\mathrm{e}^{\frac{2 \pi i r\left(s+\frac{1}{2}\right)}{q}}\left(\chi_{1, s+1 / 2}^{\text {even }}+\chi_{1, s+1 / 2}^{\text {odd }}\right)\right)\right. \\
& -\frac{\sqrt{2}}{2}\left(\mathrm{e}^{\frac{2 \pi i r s}{q}}\left(\chi_{\sigma, s}^{\text {even }}+\chi_{\sigma, s}^{\text {odd }}\right)+\mathrm{e}^{\frac{2 \pi i r\left(s+\frac{1}{2}\right)}{q}}\left(\chi_{\sigma, s+1 / 2}^{\text {even }}+\chi_{\sigma, s+1 / 2}^{\text {odd }}\right)\right) \\
& \left.+\frac{1}{2}\left(\mathrm{e}^{\frac{2 \pi i r s}{q}}\left(\chi_{\psi, s}^{\text {even }}+\chi_{\psi, s}^{\text {odd }}\right)+\mathrm{e}^{\frac{2 \pi i r\left(s+\frac{1}{2}\right)}{q}}\left(\chi_{\psi, s+1 / 2}^{\text {even }}+\chi_{\psi, s+1 / 2}^{\text {odd }}\right)\right)\right\}, \\
& \chi_{1, r}^{\text {odd }} \rightarrow \frac{\mathrm{e}^{i \frac{\pi}{q} \operatorname{Re} \frac{\zeta^{2}}{\tau}}}{2 \sqrt{q}} \sum_{s=0}^{q-1}\left\{\frac{1}{2}\left(\mathrm{e}^{\frac{2 \pi i r s}{q}}\left(\chi_{1, s}^{\text {even }}+\chi_{1, s}^{\text {odd }}\right)-\mathrm{e}^{\frac{2 \pi i r\left(s+\frac{1}{2}\right)}{q}}\left(\chi_{1, s+1 / 2}^{\text {even }}+\chi_{1, s+1 / 2}^{\text {odd }}\right)\right)\right. \\
& -\frac{\sqrt{2}}{2}\left(\mathrm{e}^{\frac{2 \pi i r s}{q}}\left(\chi_{\sigma, s}^{\text {even }}+\chi_{\sigma, s}^{\text {odd }}\right)-\mathrm{e}^{\frac{2 \pi i r\left(s+\frac{1}{2}\right)}{q}}\left(\chi_{\sigma, s+1 / 2}^{\text {even }}+\chi_{\sigma, s+1 / 2}^{\text {odd }}\right)\right) \\
& \left.+\frac{1}{2}\left(\mathrm{e}^{\frac{2 \pi i r s}{q}}\left(\chi_{\psi, s}^{\text {even }}+\chi_{\psi, s}^{\text {odd }}\right)-\mathrm{e}^{\frac{2 \pi i r\left(s+\frac{1}{2}\right)}{q}}\left(\chi_{\psi, s+1 / 2}^{\text {even }}+\chi_{\psi, s+1 / 2}^{\text {odd }}\right)\right)\right\} \\
& \chi_{\sigma, r+1 / 2}^{\text {even }} \rightarrow \frac{\mathrm{e}^{i \frac{\pi}{q} \operatorname{Re} \frac{\zeta^{2}}{\tau}}}{2 \sqrt{q}} \sum_{s=0}^{q-1}\left\{\frac{\sqrt{2}}{2}\left(\mathrm{e}^{\frac{2 \pi i\left(r+\frac{1}{2}\right) s}{q}}\left(\chi_{1, s}^{\text {even }}-\chi_{1, s}^{\text {odd }}\right)+\mathrm{e}^{\frac{2 \pi i\left(r+\frac{1}{2}\right)\left(s+\frac{1}{2}\right)}{q}}\left(\chi_{1, s+1 / 2}^{\text {even }}-\chi_{1, s+1 / 2}^{\text {odd }}\right)\right)\right. \\
& \left.-\frac{\sqrt{2}}{2}\left(\mathrm{e}^{\frac{2 \pi i\left(r+\frac{1}{2}\right) s}{q}}\left(\chi_{\psi, s}^{\text {even }}-\chi_{\psi, s}^{\text {odd }}\right)+\mathrm{e}^{\frac{2 \pi i\left(r+\frac{1}{2}\right)\left(s+\frac{1}{2}\right)}{q}}\left(\chi_{\psi, s+1 / 2}^{\text {even }}-\chi_{\psi, s+1 / 2}^{\text {odd }}\right)\right)\right\}
\end{aligned}
$$




$$
\begin{aligned}
\chi_{\sigma, r+1 / 2}^{\text {even }} & \rightarrow \frac{\mathrm{e}^{i \frac{\pi}{q} \operatorname{Re} \frac{\zeta^{2}}{\tau}}}{2 \sqrt{q}} \sum_{s=0}^{q-1}\left\{\frac{\sqrt{2}}{2}\left(\mathrm{e}^{\frac{2 \pi i\left(r+\frac{1}{2}\right) s}{q}}\left(\chi_{1, s}^{\text {even }}-\chi_{1, s}^{\text {odd }}\right)-\mathrm{e}^{\frac{2 \pi i\left(r+\frac{1}{2}\right)\left(s+\frac{1}{2}\right)}{q}}\left(\chi_{1, s+1 / 2}^{\text {even }}-\chi_{1, s+1 / 2}^{\text {odd }}\right)\right)\right. \\
& \left.-\frac{\sqrt{2}}{2}\left(\mathrm{e}^{\frac{2 \pi i\left(r+\frac{1}{2}\right) s}{q}}\left(\chi_{\psi, s}^{\text {even }}-\chi_{\psi, s}^{\text {odd }}\right)-\mathrm{e}^{\frac{2 \pi i\left(r+\frac{1}{2}\right)\left(s+\frac{1}{2}\right)}{q}}\left(\chi_{\psi, s+1 / 2}^{\text {even }}-\chi_{\psi, s+1 / 2}^{\text {odd }}\right)\right)\right\} .
\end{aligned}
$$

By using these formulas, the modular behavior of each sector in (3.31) is deduced as

$$
\begin{aligned}
& \widetilde{S}: \chi_{1, r}^{\mathrm{Pf}} \rightarrow \frac{\mathrm{e}^{i \frac{\pi}{q} \operatorname{Re} \frac{\zeta^{2}}{\tau}}}{2 \sqrt{q}} \sum_{s=0}^{q-1}\left(\mathrm{e}^{\frac{2 \pi i r s}{q}}\left(\chi_{1, r}^{\mathrm{Pf}}+\chi_{\psi, r}^{\mathrm{Pf}}\right)+\sqrt{2} \mathrm{e}^{\frac{2 \pi i r\left(s+\frac{1}{2}\right)}{q}} \chi_{\sigma, s+\frac{1}{2}}^{\mathrm{Pf}}\right), \\
& \widetilde{S}: \chi_{\psi, r}^{\mathrm{Pf}} \rightarrow \frac{\mathrm{e}^{i \frac{\pi}{q} \operatorname{Re} \frac{\zeta^{2}}{\tau}}}{2 \sqrt{q}} \sum_{s=0}^{q-1}\left(\mathrm{e}^{\frac{2 \pi i r s}{q}}\left(\chi_{1, r}^{\mathrm{Pf}}+\chi_{\psi, r}^{\mathrm{Pf}}\right)-\sqrt{2} \mathrm{e}^{\frac{2 \pi i r\left(s+\frac{1}{2}\right)}{q}} \chi_{\sigma, s+\frac{1}{2}}^{\mathrm{Pf}}\right), \\
& \widetilde{S}: \chi_{\sigma, r+1 / 2}^{\mathrm{Pf}} \rightarrow \frac{\sqrt{2} \mathrm{e}^{i \frac{\pi}{q} \operatorname{Re} \frac{\zeta^{2}}{\tau}}}{2 \sqrt{q}} \sum_{s=0}^{q-1} \mathrm{e}^{\frac{2 \pi i\left(r+\frac{1}{2}\right) s}{q}}\left(\chi_{1, r}^{\mathrm{Pf}}-\chi_{\psi, r}^{\mathrm{Pf}}\right) .
\end{aligned}
$$

Thus $\chi_{i, r}^{\text {Pf }}$ form an irreducible representation of the modular group. It is readily seen that the annulus partition function (3.31) is invariant under $(S)$.

Let us recall the fusion rules formed by quasiparticles in the Pfaffian state. They are

$$
\begin{aligned}
1 e^{i \frac{r}{\sqrt{q}}} \times 1 e^{i \frac{s}{\sqrt{q}}} & =1 e^{i \frac{r+s}{\sqrt{q}}}, \\
1 e^{i \frac{r}{\sqrt{q}}} \times \psi e^{i \frac{s}{\sqrt{q}}} & =\psi e^{i \frac{r+s}{\sqrt{q}}}, \\
1 e^{i \frac{r}{\sqrt{q}}} \times \sigma e^{i \frac{s+1 / 2}{\sqrt{q}}} & =\sigma e^{i \frac{r+s+1 / 2}{\sqrt{q}}}, \\
\psi e^{i \frac{r}{\sqrt{q}}} \times \psi e^{i \frac{s}{\sqrt{q}}} & =1 e^{i \frac{r+s}{\sqrt{q}}}, \\
\psi e^{i \frac{r}{\sqrt{q}}} \times \sigma e^{i \frac{s+1 / 2}{\sqrt{q}}} & =\sigma e^{i \frac{r+s+1 / 2}{\sqrt{q}}}, \\
\sigma e^{i \frac{r+1 / 2}{\sqrt{q}}} \times \sigma e^{i \frac{s+1 / 2}{\sqrt{q}}} & =1 e^{i \frac{r+s}{\sqrt{q}}}+\psi e^{i \frac{r+s+1}{\sqrt{q}}} .
\end{aligned}
$$

If we denote these fusion rules by $\widetilde{N_{j k}^{i}}$, it is confirmed the Verlinde formula for the Pfaffian state

$$
\begin{aligned}
\widetilde{N}_{j k}^{i} & =\sum_{n} \widetilde{S}_{j}^{n} \widetilde{\lambda}_{k}^{(n)} \widetilde{S}_{n}^{\dagger i}, \\
\widetilde{\lambda}_{k}^{(n)} & =\widetilde{S}_{k}^{n} / \widetilde{S}_{0}^{n} .
\end{aligned}
$$

This formula can be used to compute the topological order of the Pfaffian state as Cappelli and Zemba do for the abelian cases in [10]. For example, the degeneracy of the ground state of the Pfaffian state at $\nu=1 / q$ on a genus $g$ Riemann surface is given by $3^{g} q^{g}$.

\subsubsection{The Haldane-Rezayi state}

The annulus grand-canonical partition function for the Haldane-Rezayi state is given by 9]

$$
\begin{aligned}
Z_{\mathrm{HR}}^{\mathrm{ann}}=\sum_{r=0}^{q-1}\left\{2\left|\chi_{1, r+1 / 2}+\chi_{\psi, r+1 / 2}\right|^{2}\right. & \left.+\left|\chi_{\sigma, r}^{\text {even }}+\chi_{\widetilde{\sigma}, r}^{\text {odd }}\right|^{2}+\left|\chi_{\sigma, r}^{\text {odd }}+\chi_{\widetilde{\sigma}, r}^{\text {even }}\right|^{2}\right\} \\
\chi_{i, r} & =\chi_{i}^{c=-2} \chi_{r / q}, \quad i=1, \psi, \\
\chi_{\sigma, r+1 / 2}^{a} & =\chi_{\sigma}^{c=-2} \chi_{(r+1 / 2) / q}^{a}, \quad a=\text { even, odd }
\end{aligned}
$$


where $\chi_{1}^{c=-2}, \chi_{\psi}^{c=-2}, \chi_{\sigma}^{c=-2}, \chi_{\widetilde{\sigma}}^{c=-2}$ are the characters for the ones of $c=-2$ scalar fermion :

$$
\begin{aligned}
\chi_{1}^{c=-2} & =\frac{1}{2} \omega^{\frac{1}{12}}\left[\prod_{1}^{\infty}\left(1+\omega^{n}\right)^{2}+\prod_{1}^{\infty}\left(1-\omega^{n}\right)^{2}\right] \\
& =\frac{1}{2}\left(\frac{\theta_{2}(\tau)}{2 \eta(\tau)}+\eta(\tau)^{2}\right) \\
\chi_{\psi}^{c=-2} & =\frac{1}{2} \omega^{\frac{1}{12}}\left[\prod_{1}^{\infty}\left(1+\omega^{n}\right)^{2}-\prod_{1}^{\infty}\left(1-\omega^{n}\right)^{2}\right] \\
& =\frac{1}{2}\left(\frac{\theta_{2}(\tau)}{2 \eta(\tau)}-\eta(\tau)^{2}\right), \\
& =\frac{1}{2} \omega^{-\frac{1}{24}}\left(\prod_{0}^{\infty}\left(1+\omega^{n+\frac{1}{2}}\right)^{2}+\prod_{0}^{\infty}\left(1-\omega^{n+\frac{1}{2}}\right)^{2}\right) \\
\chi_{\sigma}^{c=-2} & \frac{1}{2}\left(\frac{\theta_{3}(\tau)}{\eta(\tau)}+\frac{\theta_{4}(\tau)}{\eta(\tau)}\right), \\
\chi_{\tilde{\sigma}}^{c=-2} & \frac{1}{2} \omega^{-\frac{1}{24}}\left(\prod_{0}^{\infty}\left(1+\omega^{n+\frac{1}{2}}\right)^{2}-\prod_{0}^{\infty}\left(1-\omega^{n+\frac{1}{2}}\right)^{2}\right) \\
& =\frac{1}{2}\left(\frac{\theta_{3}(\tau)}{\eta(\tau)}-\frac{\theta_{4}(\tau)}{\eta(\tau)}\right) .
\end{aligned}
$$

The factor 2 in (3.61) is from the zero modes of the scalar fermions. As noted in [39, 40], the behaviors of $\chi_{1}^{c=-2}$ and $\chi_{\psi}^{c=-2}$ under $S$ involve the coefficients which are themselves functions of $\tau$ (from $\eta^{2}$ terms). However the extended characters which appear in (3.63) are

$$
\begin{aligned}
\chi_{\Psi, r}^{\mathrm{HR}} & =\sqrt{2}\left(\chi_{1, r}+\chi_{\psi, r}\right) \equiv \sqrt{2} \chi_{\Psi}^{c=-2} \chi_{r / q}, \quad \chi_{\Psi}^{c=-2}=\chi_{1}^{c=-2}+\chi_{\psi}^{c=-2}, \\
\chi_{\sigma, r+1 / 2}^{\mathrm{HR}} & =\chi_{\sigma, r+1 / 2}^{\text {even }}+\chi_{\widetilde{\sigma}, r+1 / 2}^{\text {odd }}, \\
\chi_{\widetilde{\sigma}, r+1 / 2}^{\mathrm{HR}} & =\chi_{\sigma, r+1 / 2}^{\text {even }}+\chi_{\widetilde{\sigma}, r+1 / 2}^{\text {odd }} .
\end{aligned}
$$

Thus $\chi_{1}^{c=-2}$ and $\chi_{\psi}^{c=-2}$ only through the form $\chi_{\Psi}^{c=-2}=\chi_{1}^{c=-2}+\chi_{\psi}^{c=-2}$ and the $\tau$ dependence of the modular matrix elements disappears.

The extended characters transform under $S$ as

$$
\begin{aligned}
\widetilde{S}: \chi_{\Psi}^{\mathrm{HR}} & \rightarrow \frac{\sqrt{2} \mathrm{e}^{i \frac{\pi}{q} \operatorname{Re} \frac{\zeta^{2}}{\tau}}}{2 \sqrt{q}} \sum_{s=0}^{q-1} \mathrm{e}^{\frac{2 \pi i r s}{q}}\left(\chi_{\sigma}^{c=-2} \chi_{s / q}-\chi_{\sigma}^{c=-2} \chi_{s / q}\right) \\
\widetilde{S}: \chi_{\sigma, r+1 / 2}^{\mathrm{HR}} & \rightarrow \frac{\mathrm{e}^{i \frac{\pi}{q} \operatorname{Re} \frac{\zeta^{2}}{\tau}}}{2 \sqrt{q}} \sum_{s=0}^{q-1}\left\{\mathrm{e}^{\frac{2 \pi i\left(r+\frac{1}{2}\right) s}{q}}\left(\chi_{\sigma}^{c=-2}+\chi_{\widetilde{\sigma}}^{c=-2}\right)\left(\chi_{s / q}^{\mathrm{even}}-\chi_{s / q}^{\text {odd }}\right)\right. \\
& \left.+\sqrt{2} \mathrm{e}^{\frac{2 \pi i\left(r+\frac{1}{2}\right)\left(s+\frac{1}{2}\right)}{q}} \chi_{\Psi}^{c=-2}\left(\chi_{(s+1 / 2) / q}^{\mathrm{even}}-\chi_{(s+1 / 2) / q}^{\text {odd }}\right)\right\} \\
\widetilde{S}: \chi_{\widetilde{\sigma}, r+1 / 2}^{\mathrm{HR}} & \rightarrow \frac{\mathrm{e}^{i \frac{\pi}{q} \operatorname{Re} \frac{\zeta^{2}}{\tau}}}{2 \sqrt{q}} \sum_{s=0}^{q-1}\left\{\mathrm{e}^{\frac{2 \pi i\left(r+\frac{1}{2}\right) s}{q}}\left(\chi_{\sigma}^{c=-2}+\chi_{\widetilde{\sigma}}^{c=-2}\right)\left(\chi_{s / q}^{\mathrm{even}}-\chi_{s / q}^{\text {odd }}\right)\right. \\
& \left.-\sqrt{2} \mathrm{e}^{\frac{2 \pi i\left(r+\frac{1}{2}\right)\left(s+\frac{1}{2}\right)}{q}} \chi_{\Psi}^{c=-2}\left(\chi_{(s+1 / 2) / q}^{\text {even }}-\chi_{(s+1 / 2) / q}^{\text {odd }}\right)\right\} .
\end{aligned}
$$

The problem here is that the extended characters $\chi_{\Psi, r}^{\mathrm{HR}}, \chi_{\sigma, r+1 / 2}^{\mathrm{HR}}, \chi_{\widetilde{\sigma}, r+1 / 2}^{\mathrm{HR}}$ are not closed under the modular transformation. Thus they do not form a representation of the modular group. Accordingly $Z_{\mathrm{ann}}^{\mathrm{HR}}$ does not satisfy the modular invariance conditions. 
One method to cure this trouble is to map the edge theory by the map of $c=-2$ scalar fermion to $c=1$ Dirac fermion as in [39, 41, 29]. A simple way to accomplish it is to insert $(-1)^{F}$ ( $F$ is the number operator for fermion) in the trace. Actually this modification is already mentioned in [9]. It interchanges the untwisted and twisted sectors of the scalar fermion, and the couplings become

$$
\chi_{1}^{c=-2} \chi_{(r+1 / 2) / q}^{a}, \quad \chi_{\psi}^{c=-2} \chi_{(r+1 / 2) / q}^{a}, \quad \chi_{\sigma}^{c=-2} \chi_{r / q}^{a}, \quad \chi_{\widetilde{\sigma}}^{c=-2} \chi_{r / q}^{a} .
$$

These couplings are actually the same as the ones for the 331 state, whose internal degrees of freedom are given by the Dirac fermion. The partition function now equals to the one for the 331 state, $Z_{331}^{\mathrm{ann}}$. For the 331 state the modular invariance conditions are verified as in the Pfaffian state and the fusion rules of quasiparticles are obtained correctly by the Verlinde formula. Actually the 331 state is a part of the generalized hierarchy 42 by the equivalence of the compactified boson at $R=1$ and the Dirac fermion. Thus the modular properties also follows from [10].

However, the physical origin of insertion of $(-1)^{F}$ or other maps of the scalar fermion to the Dirac fermion is unclear. Here we give another method which can be interpreted physically. We consider a half transformation of $(V)$ of (3.4),

$$
\zeta \rightarrow \zeta+\tau / 2
$$

which we temporarily call $(X)$. Under the transformation $(X), \chi_{r / q}^{a}$ and $\chi_{(r+1 / 2) / q}^{a}$ transform as

$$
\begin{gathered}
X: \chi_{r / q}^{a} \rightarrow e^{-\frac{\pi i}{2 q}\left(2 \operatorname{Re} \zeta+\operatorname{Re} \frac{\tau}{2}\right)} \chi_{(r+1 / 2) / q}^{a}, \\
X: \chi_{(r+1 / 2) / q}^{a} \rightarrow e^{-\frac{\pi i}{2 q}\left(2 \operatorname{Re} \zeta+\operatorname{Re} \frac{\tau}{2}\right)} \chi_{(r+1) / q}^{a} .
\end{gathered}
$$

Thus, under this transformation, the couplings in the Haldane-Rezayi state are transformed to (3.78), the ones for the 331 state. For the partition function we thus establish the following identity:

$$
Z_{\mathrm{HR}}^{\mathrm{ann}}\left(\tau, \zeta+\frac{\tau}{2}\right)=Z_{331}^{\mathrm{ann}}(\tau, \zeta)
$$

The physical interpretation of $(X)$ is the addition of a half unit flux quantum through the center of the annulus, which causes a spectral flow This interpretation is transparent in the modular transformed version of (3.82):

$$
Z_{\mathrm{HR}}^{\mathrm{ann}}\left(\tau^{\prime}, \zeta^{\prime}+\frac{1}{2}\right)=Z_{331}^{\mathrm{ann}}\left(\tau^{\prime}, \zeta^{\prime}\right)
$$

where $\tau^{\prime}=-\frac{1}{\tau}$ and $\zeta^{\prime}=\frac{\zeta}{\tau}$. This identity implies that the Haldane-Rezayi state becomes a modular invariant consistent state under the presence of a half unit flux quantum. In this picture, the Haldane-Rezayi state requires such an additional flux for its physical consistency. This observation possibly gives a clue for ' The $\nu=\frac{5}{2}$ Enigma' 27]. Especially microscopic origin of the flux should be given.

Conversely, a $2+1$ dimensional effective field theory of the Haldane-Rezayi state may be realized as the effective field theory of the 331 state with an additional flux i.e. two component Chern-Simons theory with a flux of a half unit quantum 45.

$\dagger$ The similar relation was also found in the calculation of the persistent edge current of the Haldane-Rezayi state 44 . 
For the Haldane-Rezayi state, as quasiparticles involve a logarithmic operator [29], the fusion rules in the bulk obviously cannot be obtained from the modular matrix elements of the 331 state. The microscopic study 443 supports that the topological orders in the Haldane-Rezayi state is indeed given by the $c=-2$ scalar fermion. Thus the computation of the topological order from the modular matrix elements in Ref. 10 can not be extended to the Haldane-Rezayi state.

\section{Conclusions}

In this paper, we first considered the spinon-holon FQH state constructed from a conformal block of the $S U(2)$ Wess-Zumino-Witten model at level $k$ and obtained the partition functions of the edge excitations on arbitrary multiply connected domains. We showed that different sectorings for the holon edge excitations appear according to whether the level $k$ is even or odd. We then investigated the modular properties of the characters for nonabelian FQH states. We determined the modular properties of the $\mathrm{U}(1)$ characters which appear in nonabelian FQH states. From them, we derived the modular properties of the characters for the spinon-holon state and paired states. We confirmed the extended modular invariance conditions for annulus partition functions. This enabled us to extend the relation between the topological order and the modular matrix elements to nonabelian cases. We also found that the extended characters for the Haldane-Rezayi state do not form a representation of the modular group. We showed a new identity between the partition function for the Haldane-Rezayi state and the one for the 331 state and suggested the addition of half unit flux quantum through the center of annulus to obtain the physically plausible partition function for the Haldane-Rezayi state. However, the relation between the modular properties and the topological order fails in the Haldane-Rezayi state even after the modification because of the presence of a logarithmic operator in the bulk. We also suggested that the appearance of the additional flux may yield a clue for 'The $\nu=\frac{5}{2}$ Enigma'.

\section{Acknowledgement}

I would like to thank A.Cappelli, M.Flohr, D.Lidsky, J.Shiraishi and G.R.Zemba for beneficial comments and encouragement.

\section{References}

[1] For a recent review, see Perspective in Quantum Hall Effects, edited by S. Das Sarma and A.Pinczuk (John Wiley and Son, 1997).

[2] R. B. Laughlin, Phys. Rev. Lett. 50 (1983) 1395.

[3] M. Stone, Ann. Phys. (NY) 207 (1991) 38 ;Phys. Rev. B 42 (1990)8399.

[4] X.G. Wen, Phys. Rev. B41(1990) 12838; for a review see: X.G. Wen, Int. J. Mod. Phys. B6(1992)1711.

[5] C.L. Kane and M.P.A. Fisher, Phys. Rev. B46(1992)15233; K. Moon, H. Yi, C. L. Kane, S. M. Girvin and M. P. A.Fisher, Phys. Rev. Lett. 71, (1993) 4381 
[6] F.P. Milliken et al., Solid State Commun. 97(1996)309; A.M. Chang et al., Phys. Rev. Lett. 77,2538(1996) ;R. de-Picciotto et al., Nature,389(1997)162; L. Saminadayar et al., Phys. Rev. Lett. 79(1997) 2526.

[7] A. Cappelli, G.V. Dunne, C.A. Trugenberger and G.R. Zemba, Nucl. Phys. B398(1993) 531.

[8] X.G. Wen and Y.S. Wu, Nucl. Phys. B419 (1994)455; X.G. Wen, Y.S. Wu and Y.Hatsugai, Nucl. Phys. B422[FS] (1994)476.

[9] M. Milovanović and N. Read, Phys. Rev. B53(1996)13559.

[10] A. Cappelli and G.R. Zemba, Nucl. Phys. B490 (1997) 595.

[11] X.-G. Wen, Phys. Rev. 40 (1989) 7387.

[12] J.K. Jain, Phys. Rev. B40(1989)8079.

[13] T. Gannon, Nucl. Phys. B491(1997)659.

[14] G. Moore and N. Read, Nucl. Phys. B360 (1991) 362.

[15] F.D.M. Haldane and E.H. Rezayi, Phys. Rev. Lett. 60 (1988) 956.

[16] B. Blok and X.G. Wen, Nucl. Phys. B374 (1992) 615.

[17] J. Fröhlich and U.M. Studer, Rev. Mod. Phys. 65(1993) 733; C. Nayak and F. Wilczek, Nucl. Phys. B450(1995)58, Nucl. Phys. B455(1995)493.

[18] A. De Martino and R. Musto, Int. J. Mod. Phys. B9(1995)2839.

[19] D. C. Cabra, G.L. Rossini Mod. Phys. Lett. A12 (1997) 265-276.

[20] B.I. Halperin, Helv.Phys.Acta. 56(1983)75.

[21] A. Balatsky and M. Stone, Phys. Rev. B43(1991)8038; A. Balatsky and E. Fradkin, Phys. Rev. B43(1991)10622.

[22] C. Cristofano, G. Mauekka, R. Musto, F. Nicodemi, Phys.Lett.B262(1991)88; S. Fubini, Mod. Phys. Lett. A6(1991)347; M. Flohr, Mod. Phys. Lett. A11(1996)55.

[23] E. Witten, Commun.Math.Phys. B121(1989) 351; G. Moore and N. Seiberg, Phys. Lett. B220 (1989) 422; S. Elitzur, G. Moore, A. Schwimmer and N. Seiberg, Nucl. Phys. B326 (1989) 108; G. Dunne, R. Jackiw and C. Trugenberger, Ann. Phys. (NY) 194 (1989) 197.

[24] R.L.Willet, J. P. Eisenstein, H. L. Stormer, D.C.Tsui, A.C.Gossard and J.H.English, Phys. Rev. Lett. 59 (1987) 1776.

[25] S. He, X.-C.Xie, S.Das Sarma, and F.-C.Zhang, Phys. Rev. B43(1991)9339 ; S. He, S.Das Sarma, and X.-C.Xie, ibid. 47(1993)4394 .

[26] R.H.Morf, Phys. Rev. Lett. 80(1998)1505.

[27] J.Eisenstein, Experimental Studies of Multicomponent Quantum Hall Systems in [1]. 
[28] C. Nayak and F. Wilczek, Nucl. Phys. B479(1996)529.

[29] V. Gurarie, M. Flohr and C. Nayak, Nucl. Phys. B498 (1997) 513.

[30] E. Fradkin, C. Nayak, A. Tsvelik and F. Wilczek, Nucl. Phys. 556(1998)704.

[31] K. Schoutens, cond-mat/9803169.

[32] A. Cappelli, C. Itzykson and J. Zuber, Comm. Math. Phys. 113(1987)1. ; A. Kato, Mod. Phys. Lett. A2(1987)585.

[33] K. Ino, cond-mat/9711016, to appear in Int. J. Mod. Phys. A.

[34] J.C. Lee and X.G. Wen, cond-mat/9705033.

[35] G. Moore and N. Seiberg, Phys. Lett. B212 (1989) 451 ; Nucl. Phys. B313 (1989) 16 ; Comm. Math. Phys. 123 (1989) 77.

[36] E. Verlinde, Nucl. Phys. B300[FS22] (1988) 360.

[37] D. Gepner and E. Witten, Nucl. Phys. B278(1986) 493.

[38] P. Bouwknegt, A. Ludwig, and K. Schoutens, Phys. Lett. B359 (1995) 304.

[39] M. Flohr, Int. J. Mod. Phys. A11 (1996) 4147.

[40] M. R. Gaberdiel and H. G. Kausch, Phys. Lett. B386 (1996) 131.

[41] S. Guruswamy and A.W.W. Ludwig, hep-th/9612172.

[42] J. Fröhlich and A. Zee, Nucl. Phys. B364 (1991) 517 ; X.-G. Wen and A. Zee, Phys. Rev. B46 (1993) 2290.

[43] N. Read and E.H. Rezayi, Phys. Rev. B54(1996)16864.

[44] K. Ino, Phys. Rev. Lett. 81(1998)1078.

[45] K. Ino, in preparation. 